\title{
Exotic dynamics in a firing rate model of neural tissue with threshold accommodation
}

\author{
S Coombes and M R Owen
}

\begin{abstract}
Many of the equations describing the dynamics of neural systems are written in terms of firing rate functions, which themselves are often taken to be threshold functions of synaptic activity. Dating back to work by Hill in 1936 it has been recognized that more realistic models of neural tissue can be obtained with the introduction of state-dependent dynamic thresholds. In this paper we treat a specific phenomenological model of threshold accommodation that mimics many of the properties originally described by Hill. Importantly we explore the consequences of this dynamic threshold at the tissue level, by modifying a standard neural field model of Wilson-Cowan type. As in the case without threshold accommodation classical Mexican-Hat connectivity is shown to allow for the existence of spatially localized states (bumps) in both one and two dimensions. Importantly an analysis of bump stability in one dimension, using recent Evans function techniques, shows that bumps may undergo instabilities leading to the emergence of both breathers and traveling waves. Moreover, a similar analysis for traveling pulses leads to the conditions necessary to observe a stable traveling breather. In the regime where a bump solution does not exist direct numerical simulations show the possibility of self-replicating bumps via a form of bump splitting. Simulations in two space dimensions show analogous localized and traveling solutions to those seen in one dimension. Indeed dynamical behavior in this neural model appears reminiscent of that seen in other dissipative systems that support localized structures, and in particular those of coupled cubic complex Ginzburg-Landau equations. Further numerical explorations illustrate that the traveling pulses in this model exhibit particle like properties, similar to those of dispersive solitons observed in some three component reaction-diffusion systems.

A preliminary account of this work first appeared in S Coombes and M R Owen, Bumps, breathers, and waves in a neural network with spike frequency adaptation, Physical Review Letters 94 (2005), 148102(1-4).
\end{abstract}

\section{Introduction}

The mathematical modeling of neural tissue can trace its roots back to work by Beurle 4 in the 1950s and later by Griffith $[\mathbf{2 0}, \mathbf{2 1}$ in the 1960s. To overcome the difficulties of modeling the large numbers of neurons and synapses in even

2000 Mathematics Subject Classification. 45G10; 92C20.

Key words and phrases. neural, breather, threshold accommodation, Evans function. Supported by EPSRC Grant No. GR/R76219. 
a small piece of cortex these authors advocated continuum descriptions in which space is continuous and macroscopic state variables are mean firing rates. These early models were later improved and refined in the work of Wilson and Cowan [48, Nunez [38 and Amari [2], and are still in use today as models of large scale brain activity. We shall refer to such models and their variants as neural field theories. To date neural field theories have found applications in understanding brain slice preparations [26, EEG rhythms [31, 34, visual hallucinations [15, 7, short term memory [32, motion perception [19], representations in the headdirection system [50] and feature selectivity in the visual cortex [3]. For recent reviews of the dynamics of neural fields we refer the reader to [9, 10. Typically they take the form of integral or integro-differential equations. Although they do not capture the details of the fast ionic currents underlying spiking neural behavior, neural field theories can incorporate realistic non-local axo-dendritic synaptic interactions provided that spike-trains can be replaced by firing rates. In practice this assumption seems to be useful when dealing with slow synaptic interactions. The firing rate in such models is often taken to be a threshold function of the synaptic activity. Typically, as a constant current is increased, most cortical neurons switch from a resting constant potential to an active mode. In the active mode, either trains of spikes are generated or bursts of spikes. Since the majority of cells in cortical networks fire repetitively [47, we shall focus our attention on this case. A common choice to fit the firing rate function is a sigmoid function of pre-synaptic activity $u$ in the form $f=f(u-h)$, where

$$
f(u)=\frac{1}{1+\exp (-\beta u)} .
$$

Here the parameter $h$ is identified as a threshold whilst $\beta$ measures the steepness of the sigmoid. Interestingly the notion of a threshold goes back a long way in the history of neuroscience. Even in the absence of any detailed model of neural tissue Hill [25] in 1936 described the fruitfulness of thinking of neural tissue as possessing a threshold for excitability. Indeed, at the single neuron level the notion of a threshold model can be traced back as far as 1907 to Lapicque [33.

In more detail we will discuss a generic neural field model with synaptic activity $u=u(x, t), x \in \mathbb{R}, t \in \mathbb{R}^{+}$, governed by the integral equation

$$
u=\eta * w \otimes f(u-h) .
$$

Here, the symbol $*$ represents a temporal convolution in the sense that

$$
(\eta * f)(x, t)=\int_{0}^{t} \eta(s) f(x, t-s) \mathrm{d} s,
$$

and $\otimes$ represents a spatial convolution such that

$$
(w \otimes f)(x, t)=\int_{-\infty}^{\infty} w(y) f(x-y, t) \mathrm{d} y .
$$

The function $\eta(t)$ (with $\eta(t)=0$ for $t<0$ ) represents a synaptic filter, whilst $w(x)$ is a synaptic footprint describing the anatomy of network connections. Typically, however, neural field theories such as the one above do not incorporate any of the slow intrinsic processes known to modulate tissue response. In particular we are drawn to the observation of Hill that thresholds are dependent upon the state of the tissue. We quote directly from his 1936 paper ${ }^{1}$ [25:

.... The critical value of $V$ required for excitation, i.e., the threshold $U$, might have

\footnotetext{
${ }^{1}$ We are indebted to John Rinzel for bringing the work of A V Hill to our attention.
} 
been constant and independent of the previous history of the nerve. If the current lasts only for a very short time this is true. If, however, the current lasts longer, the threshold rises, as is shown by the well-known fact that a slowly increasing current has a higher threshold than a quickly increasing one. The change of threshold is gradual, it takes place as a consequence of, and at a speed determined by, the change of "local potential" produced in the nerve by the passage of current. There is, therefore, a second time-factor in electric excitation, viz., that defining the rate of change of threshold $U$.

We shall use the term "accommodation" to describe the fact that the threshold U rises when the "local potential" V is maintained. It is known that "accommodation" disappears of itself, i.e., U reverts gradually to its original value $U_{0}$ when the nerve is allowed to return to its original resting state...

If we identify the "local potential" described by Hill with synaptic activity $u$ then a simple phenomenological model of threshold accommodation can be written as

$$
h_{t}=-\left(h-h_{0}\right)+\kappa g(u-\theta) .
$$

Here $h$ and $h_{0}$ are identified with the $U$ and $U_{0}$ described by Hill, whilst the nonlinear function $g(u)$ describes the effects of the accommodation process on the threshold evolution. We also assume that accommodation is itself a threshold process and interpret $\theta$ as a threshold for accommodation, whilst the parameter $\kappa>0$ measures the strength of accommodation. For later convenience we write the dynamics for $h$ in the integrated form

$$
h=h_{0}+\kappa \eta_{h} * g(u-\theta),
$$

where $\eta_{h}(t)=\mathrm{e}^{-t}$ for $t>0$ and is zero otherwise. In the absence of threshold accommodation (i.e. with $\kappa=0$ ) it is known that models with short-range excitation and long range inhibition, or so-called Mexican-hat connectivity, can support spatially localized solutions. Indeed such solutions are known to exist for sufficiently steep sigmoids 29 and has motivated much analysis in the limit that the firing rate function $f$ approaches a Heaviside step function, i.e. as $\beta \rightarrow \infty$ in (1). To illustrate how the form of nonlinear threshold accommodation (5) can lead to exotic dynamics at the network level, we will focus on the case of Mexican-hat connectivity with Heaviside firing rate function. Moreover, so that we may study instabilities of spatially localized states in a tractable mathematical fashion we will also restrict our attention to the case that $g(u)$ is also a Heaviside. Hence, throughout the rest of this paper we work with the choice $g(u)=f(u)=H(u)$, where $H$ is a Heaviside function such that $H(x)=1$ for $x \geq 0$ and is zero otherwise. Moreover, we shall take $\eta(t)=\alpha \mathrm{e}^{-\alpha t} H(t)$ and $w(x)=(1-|x|) \mathrm{e}^{-|x|}$, representing an exponential synaptic response in a network with short-range excitation and long-range inhibition. The extension to other synaptic filters and footprints is straightforward [11. A preliminary account of the work in this paper first appeared in $\mathbf{1 3}$. 


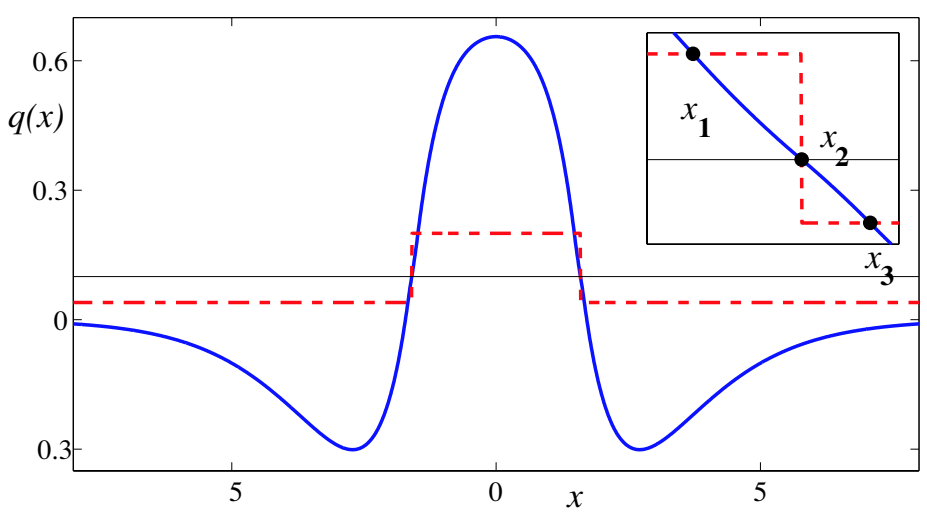

Figure 1. Analytical bump solution $(q, p)$ (as solid and dashed lines respectively) with $h_{0}=0.04 \theta=0.1, \kappa=0.16$. Here $x_{1}=$ $1.48, x_{2}=1.60$ and $x_{3}=1.67$. The inset shows a blowup of the solution around the window containing the points $x_{1}, x_{2}$ and $x_{3}$. For these values of $h_{0}$ and $\theta$ this type of solution exits for $\kappa<0.32$ (see Fig. 2).

\section{Spatially localized solutions}

First we construct time-independent solutions $(u, h)=(q(x), p(x))$ that satisfy

$$
q=w \otimes H(q-p), \quad p=\left\{\begin{array}{ll}
h_{0} & q<\theta \\
h_{0}+\kappa & q \geq \theta
\end{array} .\right.
$$

A localized bump solution is one that satisfies $q(x)>h_{0}+\kappa$ for $x \in\left[0, x_{1}\right]$, $\theta<q(x)<h_{0}+\kappa$ for $x \in\left(x_{1}, x_{2}\right), h_{0}<q(x) \leq \theta$ for $x \in\left[x_{2}, x_{3}\right)$ and $q(x)<h_{0}$ otherwise. We restrict attention to symmetric solutions for which $q(x)=q(-x)$ with $x_{3}>x_{2}>x_{1}>0$. An explicit solution may be constructed as

$$
q(x)=\left(\int_{-x_{3}}^{-x_{2}}+\int_{-x_{1}}^{x_{1}}+\int_{x_{2}}^{x_{3}}\right) w(x-y) \mathrm{d} y .
$$

The unknowns $x_{1}, x_{2}$, and $x_{3}$ are found by the simultaneous solution of

$$
q\left(x_{1}\right)=h_{0}+\kappa, \quad q\left(x_{2}\right)=\theta, \quad q\left(x_{3}\right)=h_{0} .
$$

The integrals in (8) may be evaluated to give

$$
\begin{aligned}
& q(x)=g\left(x+x_{1}\right)-g\left(x+x_{2}\right)+g\left(x+x_{3}\right) \\
&+g\left(x_{1}-x\right) H\left(x_{1}-x\right)-g\left(x-x_{1}\right) H\left(x-x_{1}\right) \\
&+\left[g\left(x_{3}-x\right)-g\left(x_{2}-x\right)\right] H\left(x_{2}-x\right)+\left[g\left(x-x_{2}\right)-g\left(x-x_{3}\right)\right] H\left(x-x_{3}\right) \\
&10) \quad+\left[g\left(x-x_{2}\right)+g\left(x_{3}-x\right)\right] H\left(x-x_{2}\right) H\left(x_{3}-x\right),
\end{aligned}
$$

where $g(x)=x \mathrm{e}^{-x}$. A plot of an analytical bump solution constructed in this fashion is shown in Fig. 1. It appears that for $\kappa$ less than some critical value there is only ever one solution of this type (see Fig. 2). 


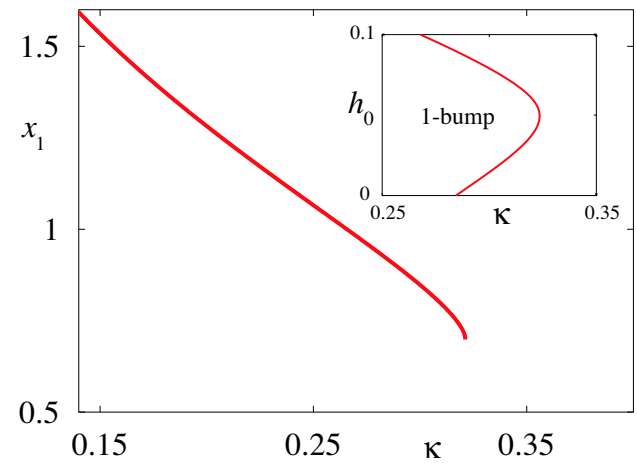

FigurE 2. Existence regions for a 1-bump solution. The main figure shows a plot of $x_{1}$ vs $\kappa$ for $\theta=0.1$ and $h_{0}=0.04$. For these parameters the 1-bump solution exists for $\kappa<\kappa_{c} \approx 0.32$. The inset show the region in $\left(\kappa, h_{0}\right)$ parameter space where 1-bump solutions exist (obtained by numerical continuation of the right most point of the main figure).

2.1. Evans function for a spatially localized solution. To assess the linear stability of this solution we use a recent Evans function approach. This was originally formulated by Evans [16 in the context of a stability theorem about excitable nerve axon equations of Hodgkin-Huxley type. The zeros of this complex analytic function determine the normal spectrum of the operator obtained by linearizing a system about its traveling wave solution (and we may view a bump solution as a stationary wave). The extension to neural field models is more recent and, for the special case of a Heaviside firing rate function, several models have now been studied $[\mathbf{9}, \mathbf{1 2}, \mathbf{1 7}, \mathbf{4 1}, \mathbf{5 1}$. Note that we are concerned with systems where the real part of the continuous spectrum has a uniformly negative upper bound, so that it is enough to determine the location of the isolated spectrum for wave stability. Recent work by Sandstede has now established, for these models, that linear stability implies nonlinear stability [44].

In more detail, we assess the linear stability of a bump solution by considering perturbations of the form $u(x, t)=q(x)+\delta u(x, t)$, and $h(x, t)=p(x)+\delta h(x, t)$. An expansion of (2) and (6) and working to first order generates the pair of equations

$$
\begin{aligned}
& \delta u=\eta * w \otimes H^{\prime}(q-p)[\delta u-\delta h], \\
& \delta h=\eta_{h} * \kappa H^{\prime}(q-\theta) \delta u .
\end{aligned}
$$

Here $H^{\prime}$ is the derivative of $H$, i.e. $H^{\prime}(x)=\delta(x)$. For perturbations of the form $(\delta u(x, t), \delta h(x, t))=(u(x), h(x)) \mathrm{e}^{\lambda t}$ we have that

$$
\frac{u}{\mathcal{L}[\eta](\lambda)}=w \otimes H^{\prime}(q-p)\left[1-\kappa \mathcal{L}\left[\eta_{h}\right](\lambda) H^{\prime}(q-\theta)\right] u,
$$

where we have eliminated the equation for $h$ using $(12)$ and introduced the Laplace transform $\mathcal{L}[\eta](\lambda)=\int_{0}^{\infty} \mathrm{d} s \mathrm{e}^{-\lambda s} \eta(s)$. Making use of the fact that

$$
\delta(q(x)-p(x))=\sum_{y= \pm x_{1}, \pm x_{3}} \frac{\delta(x-y)}{\left|q^{\prime}(y)\right|}
$$


and

$$
\delta(q(x)-p(x)) \delta(q(x)-\theta)=\frac{1}{\kappa} \sum_{y= \pm x_{2}} \frac{\delta(x-y)}{\left|q^{\prime}(y)\right|},
$$

means that the 13 takes the form

$$
\frac{u(x)}{\mathcal{L}[\eta](\lambda)}=\sum_{j=1}^{6} A_{j}(x, \lambda) u_{j},
$$

where $u_{j}=u\left(x_{j}\right)$ and $\left(x_{4}, x_{5}, x_{6}\right)=-\left(x_{1}, x_{2}, x_{3}\right), A_{1}(x, \lambda)=w\left(x-x_{1}\right) /\left|q^{\prime}\left(x_{1}\right)\right|=$ $A_{4}(-x, \lambda), A_{2}(x, \lambda)=-\mathcal{L}\left[\eta_{h}\right](\lambda) w\left(x-x_{2}\right) /\left|q^{\prime}\left(x_{2}\right)\right|=A_{5}(-x, \lambda)$, and $A_{3}(x, \lambda)=$ $w\left(x-x_{3}\right) /\left|q^{\prime}\left(x_{3}\right)\right|=A_{6}(-x, \lambda)$. The derivative of $q$ is easily calculated from (8) as $q^{\prime}(x)=W(x)-W(-x)$, where $W(x)=w\left(x+x_{1}\right)-w\left(x+x_{2}\right)+w\left(x+x_{3}\right)$. Demanding that the perturbations at $x_{j}$ be non-trivial generates an eigenvalue problem of the form $\mathcal{E}(\lambda)=0$, where $\mathcal{E}(\lambda)=\left|\mathcal{L}[\eta](\lambda)^{-1} I_{6}-\mathcal{A}(\lambda)\right|, I_{n}$ is the $n \times n$ identity matrix and $\mathcal{A}(\lambda)$ has components

$$
\mathcal{A}(\lambda)_{i j}=A_{j}\left(x_{i}, \lambda\right), \quad i, j=1, \ldots, 6 .
$$

We identify $\mathcal{E}(\lambda)$ as the Evans function for the bump, such that solutions are stable if $\operatorname{Re} \lambda<0$. The Evans function is real-valued if $\lambda$ is real. Furthermore, (i) the complex number $\lambda$ is an eigenvalue of 13 if and only if $\mathcal{E}(\lambda)=0$, and (ii) the algebraic multiplicity of an eigenvalue is equal to the order of the zero of the Evans function. For a further discussion of how to establish these results we refer the reader to $\left[\mathbf{1 2}\right.$. Using the fact that $\mathcal{L}\left[\eta_{h}\right](0)=1=\mathcal{L}[\eta](0)$ a direct calculation shows that $\mathcal{E}(0)=0$ (with corresponding eigenfunction $q^{\prime}(x)$ ), as expected for a system with translation invariance. By determining the zeros of the Evans function we are now in a position to probe the manner in which a bump may go unstable. One natural way to find the zeros of $\mathcal{E}(\lambda)$ is to write $\lambda=\nu+\mathrm{i} \omega$ and plot the zero contours of $\operatorname{Re} \mathcal{E}(\lambda)$ and $\operatorname{Im} \mathcal{E}(\lambda)$ in the $(\nu, \omega)$ plane. The Evans function is zero where the zero contours intersect. There are basically two different routes to instability, which we shall refer to as drift and breathing instability.

For sufficiently small $\kappa$ an eigenvalue crosses to the right hand complex plane on the real axis, and one sees a bump go unstable in favor of a traveling pulse with increasing $\alpha$ (where $\alpha$ controls the speed of the synapse). We illustrate this scenario with the aid of Figs. 3 and 4 . In Fig. 3 we show a sequence of plots for the Evans function for a localized bump solution with increasing $\alpha$. Figure 4 shows the result of a direct numerical simulation of the full integral model just beyond the instability point predicted from Fig. 3. We shall term the emergence of a traveling pulse from a stationary bump a drift instability and note that it can be succinctly defined by the condition that the Evans function have a repeated real root at the origin, namely $\mathcal{E}(0)=0=\mathcal{E}^{\prime}(0)$. The drift bifurcation point is then naturally defined as the least value of $\alpha$ for which $\mathcal{E}^{\prime}(0)=0$ (and being careful to also establish that there are no zeros of the Evans function in the right hand complex plane). Using Jacobi's formula for the differential of a determinant we may write

$$
\mathcal{E}^{\prime}(\lambda)=\operatorname{Tr}\left((\operatorname{adj} E(\lambda)) \frac{\mathrm{d} E}{\mathrm{~d} \lambda}\right),
$$

where $E(\lambda)=\mathcal{L}[\eta](\lambda)^{-1} I_{6}-\mathcal{A}(\lambda)$. Note that for $\lambda=0$, $\operatorname{det} E(0)=\mathcal{E}(0)=0$ and $E(0)$ is not invertible. Hence, for practical calculations we consider the limit 

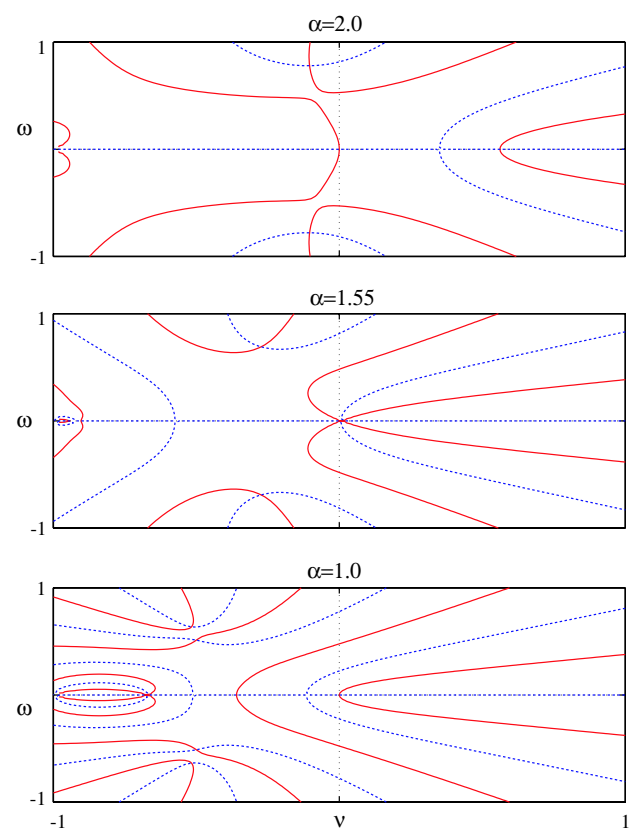

Figure 3. A plot of the Evans function for a localized bump solution with parameters as in Fig. 11. Zeros of the Evans function occur at the intersection of the solid and dashed lines where $\operatorname{Re} \mathcal{E}(\lambda)=0=\operatorname{Im} \mathcal{E}(\lambda)$. As $\alpha$ increases through $\alpha_{c} \sim 1.55$ an eigenvalue crosses to the the right hand complex plane along the real axis, signaling the onset of an instability.

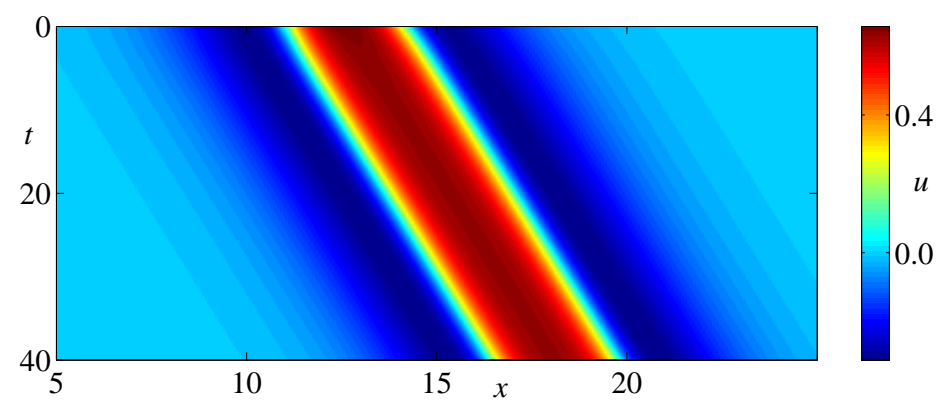

FIgURE 4. A space-time plot showing an example of a traveling pulse for $\alpha=1.6$, seen just after the point of a drift instability at $\alpha_{c} \sim 1.55$, as determined from Fig. 3 .

$\lambda \rightarrow 0^{-}$. In this case $E(\lambda)$ is invertible and we may use the result adj $E(\lambda)=$ $\mathcal{E}(\lambda) E^{-1}(\lambda)$, to establish a necessary condition for a drift bifurcation as

$$
\operatorname{Tr}\left(\left.E^{-1}\left(0^{-}\right) \frac{\mathrm{d} E}{\mathrm{~d} \lambda}\right|_{\lambda=0^{-}}\right)=0 .
$$



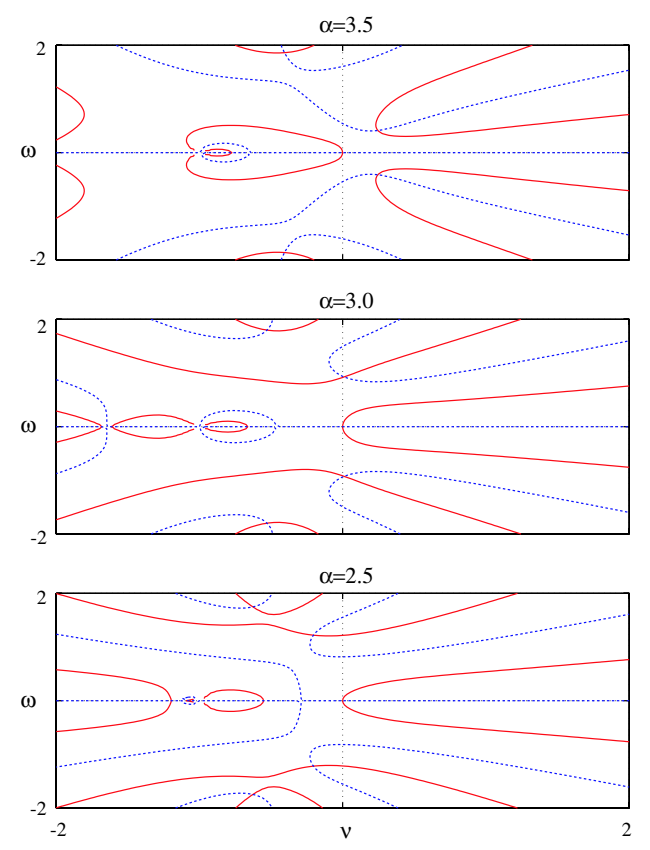

Figure 5. A plot of the Evans function for a localized bump solution at $\kappa=0.3$, and other parameters as in Fig. 1. As $\alpha$ increases through $\alpha_{c} \sim 3.0$ an eigenvalue crosses to the the right hand complex plane through the imaginary axis, signaling the onset of a dynamic instability.

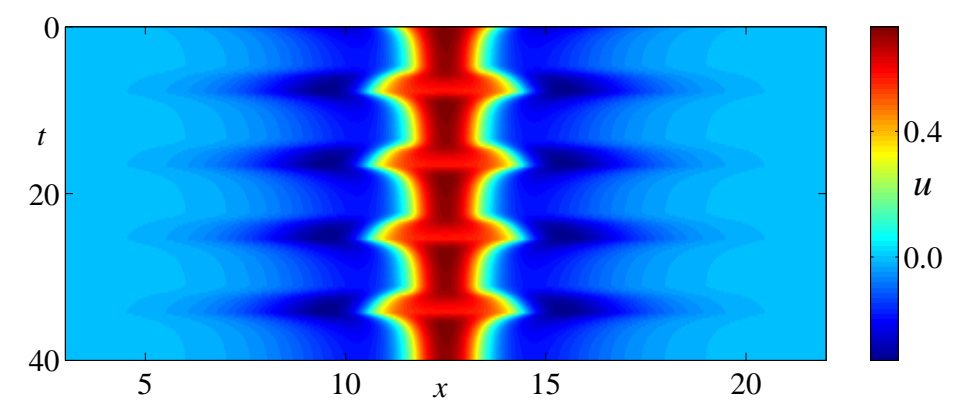

FiguRE 6. A space-time plot showing an example of a breathing solution seen for $\alpha=3.0$, just after the point of a breathing instability, as determined from Fig. 5 .

For larger $\kappa$ a pair of complex-conjugate eigenvalues cross through the imaginary axis to the right hand complex plane and a bump goes unstable in favor of a breathing solution, with increasing $\alpha$. We illustrate this scenario with the aid of Figs. 5 and 6. In Fig. 7 we present a diagram summarizing the two instabilities of a stationary bump. The drift instability border is calculated according to equation (19), whilst the breathing instability is calculated by a direct examination of plots such as shown in Fig. 5 


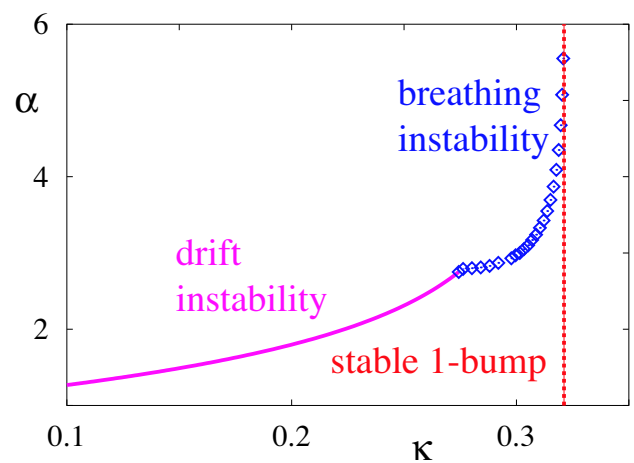

Figure 7. Instabilities of a stationary bump as a function of $\kappa$ (the strength of threshold accommodation) and $\alpha$ (the rate of synaptic decay). To the right of the vertical dashed line 1-bump solutions do not exist.

Till now we have focused our attention on so-called 1-bump solutions. However, it is possible that other forms of spatially localized $N$-bump solutions also exist. To illustrate how one may construct and analyze such solutions we briefly treat the case of a 2-bump.

2.2. Two-bump solutions. In a similar fashion to Section 2 we can write a 2-bump solution (symmetric about the origin) in the form $q(x)=W_{+}(x)+W_{-}(x)$, where

$$
W_{ \pm}(x)=\left(\int_{x_{1}}^{x_{2}}+\int_{x_{3}}^{x_{4}}+\int_{x_{5}}^{x_{6}}\right) w(y \pm x) \mathrm{d} y
$$

and $q(x) \geq p(x)$ for $x \in \pm\left[x_{1}, x_{2}\right] \bigcup\left[x_{3}, x_{4}\right] \bigcup\left[x_{5}, x_{6}\right]$. For our choice of synaptic footprint a short calculation gives

$$
\begin{aligned}
W_{+}(x) & =g\left(x+x_{2}\right)+g\left(x+x_{4}\right)+g\left(x+x_{6}\right) \\
& -g\left(x+x_{1}\right)-g\left(x+x_{3}\right)-g\left(x+x_{5}\right), \\
W_{-}(x) & =W_{1,2}(x)+W_{3,4}(x)+W_{5,6}(x),
\end{aligned}
$$

with

$$
W_{a, b}(x)=\int_{x_{a}}^{x_{b}} w(y-x) \mathrm{d} y= \begin{cases}g\left(x_{b}-x\right)-g\left(x_{a}-x\right) & x \leq x_{a} \\ g\left(x-x_{a}\right)+g\left(x_{b}-x\right) & x_{a}<x<x_{b} . \\ g\left(x-x_{a}\right)-g\left(x-x_{b}\right) & x \geq x_{b}\end{cases}
$$

The self-consistent 2-bump solution is then specified by the simultaneous solution of the six equations:

$$
q\left(x_{1}\right)=h_{0}=q\left(x_{6}\right), \quad q\left(x_{2}\right)=\theta=q\left(x_{5}\right), \quad q\left(x_{3}\right)=h_{0}+\kappa=q\left(x_{4}\right) .
$$

After enforcing these constraints it is a simple matter to prove that $q(0)=0$ for the 2-bump solution. A plot of a 2-bump is shown in Fig. 8 .

Proceeding analogously as for the 1-bump solution we can write the Evans function for a 2-bump in terms of the $12 \times 12$ matrix $\mathcal{A}(\lambda)$ with components $\mathcal{A}(\lambda)_{i j}=A_{j}\left(x_{i}, \lambda\right), \quad i, j=1, \ldots, 12$. Here, $\left(x_{7}, \ldots, x_{12}\right)=-\left(x_{1}, \ldots, x_{6}\right)$ and $A_{1}(x, \lambda)=w\left(x-x_{1}\right) /\left|q^{\prime}\left(x_{1}\right)\right|=A_{7}(-x, \lambda), A_{2}(x, \lambda)=-\mathcal{L}\left[\eta_{h}\right](\lambda) w(x-$ $\left.x_{2}\right) /\left|q^{\prime}\left(x_{2}\right)\right|=A_{8}(-x, \lambda), A_{3}(x, \lambda)=w\left(x-x_{3}\right) /\left|q^{\prime}\left(x_{3}\right)\right|=A_{8}(-x, \lambda), A_{4}(x, \lambda)=$ 


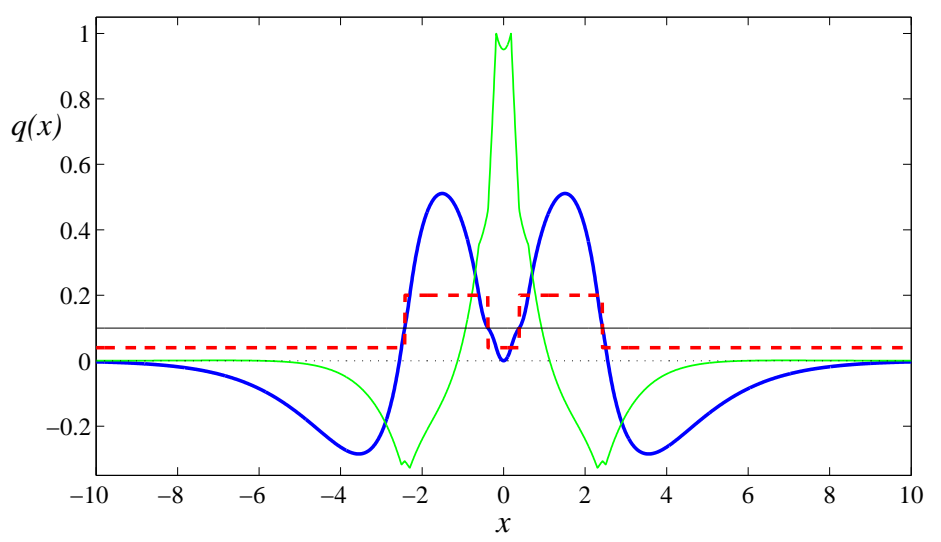

Figure 8. A 2-bump solution. Parameters as in Fig. 1 (and the 2-bump exists for $\kappa<0.1755$ ). Note that for all 2-bump solutions it may be shown that $q(0)=0$. A direct examination of the associated Evans function shows that this 2-bump is always unstable (with real eigenvalue). The green curve shows the shape of the dominant unstable eigenfunction for $\alpha=1.0$.

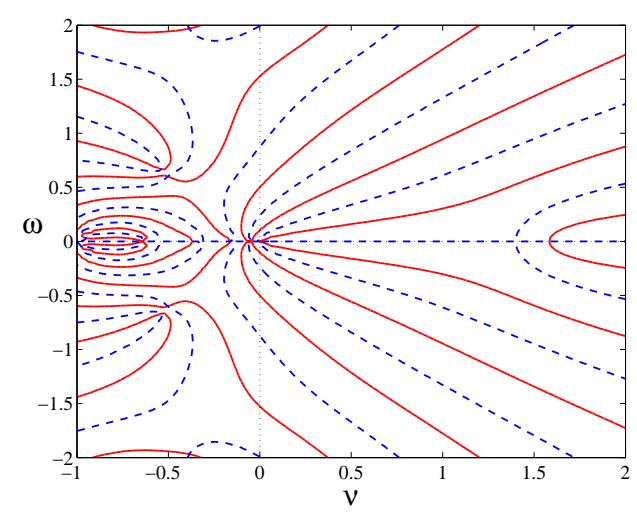

Figure 9. A plot of the Evans function for the 2-bump solution of Fig. 8 when $\alpha=1.0$, showing that the dominant eigenvalue is real and positive.

$w\left(x-x_{4}\right) /\left|q^{\prime}\left(x_{4}\right)\right|=A_{10}(-x, \lambda), A_{5}(x, \lambda)=w\left(x-x_{5}\right) /\left|q^{\prime}\left(x_{5}\right)\right|=A_{11}(-x, \lambda)$, and $A_{6}(x, \lambda)=w\left(x-x_{6}\right) /\left|q^{\prime}\left(x_{6}\right)\right|=A_{12}(-x, \lambda)$. The derivative of $q$ is easily calculated as $q^{\prime}(x)=W(x)-W(-x)$, where $W(x)=w\left(x+x_{2}\right)-w\left(x+x_{1}\right)+w\left(x+x_{4}\right)-$ $w\left(x+x_{3}\right)+w\left(x+x_{6}\right)-w\left(x+x_{5}\right)$.

A numerical examination of the Evans function suggests that the 2-bump is always unstable, as illustrated in Fig. 9. The dominant unstable mode corresponds to a real eigenvalue, with eigenfunction shown in Fig. 8. Typically, this instability of a 2-bump generates a pair of pulses traveling in opposite directions, as illustrated in Fig. 10 . 


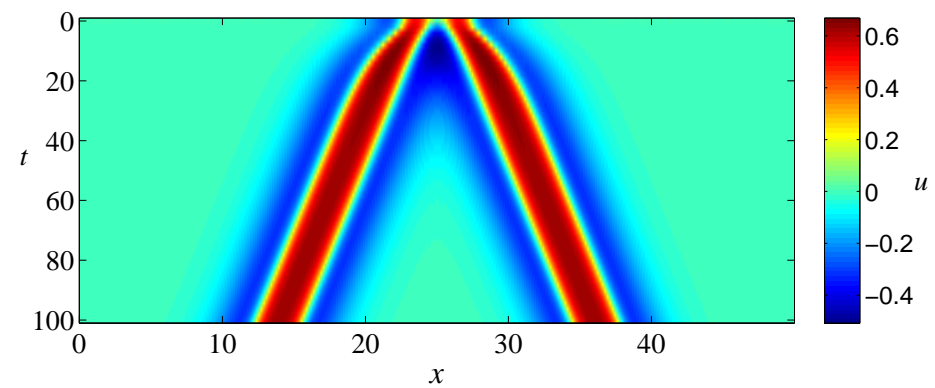

FigURE 10. A 2-bump solution is unstable (with real eigenvalue) and splits into a pair of pulses traveling in opposite directions. Parameters as in Fig. 1 1 with $\alpha=1.0$.

\section{Traveling pulses}

We have seen in Section 2.1 that a bump can destabilize in favor of a traveling pulse. It is thus natural to pursue the construction and stability analysis of traveling waves. Following the standard approach for constructing traveling wave solutions to PDEs, such as reviewed by Sandstede [43, we introduce the coordinate $\xi=x-c t$ and seek functions $\widetilde{u}(\xi, t)=u(x-c t, t)$ and $\widetilde{h}(\xi, t)=h(x-c t, t)$ that satisfy (2) and (6). In the $(\xi, t)$ coordinates we have that

$$
\begin{aligned}
\widetilde{u}(\xi, t)= & \int_{-\infty}^{\infty} \mathrm{d} y w(y) \int_{0}^{\infty} \mathrm{d} s \eta(s) \\
& \times H(\widetilde{u}(\xi-y+c s, t-s)-\widetilde{h}(\xi-y+c s, t-s)), \\
\widetilde{h}(\xi, t)= & h_{0}+\kappa \int_{0}^{\infty} \mathrm{d} s \eta_{h}(s) H(\widetilde{u}(\xi+c s, t-s)-\theta) .
\end{aligned}
$$

The traveling wave is a stationary solution $(\widetilde{u}(\xi, t), \widetilde{h}(\xi, t))=(q(\xi), p(\xi))$ (independent of $t$ ), that satisfies

$$
\begin{aligned}
& q(\xi)=\int_{0}^{\infty} \mathrm{d} s \eta(s) \psi(\xi+c s), \\
& \psi(\xi)=\int_{-\infty}^{\infty} \mathrm{d} y w(y) H(q(\xi-y)-p(\xi-y)), \\
& p(\xi)=h_{0}+\kappa \int_{0}^{\infty} \mathrm{d} s \eta_{h}(s) H(q(\xi+c s)-\theta) .
\end{aligned}
$$

We now consider traveling pulse solutions of the form $q(\xi) \geq \theta$ for $\xi \in\left[\xi_{1}, \xi_{3}\right]$ and $q(\xi)<\theta$ otherwise, as illustrated in Fig. 11. In this case the solution for $p(\xi)$ is easily calculated from 290 as

$$
p(\xi)=h_{0}+\kappa \begin{cases}{\left[1-\mathrm{e}^{-\left(\xi_{3}-\xi_{1}\right) / c}\right] \mathrm{e}^{\left(\xi-\xi_{1}\right) / c}} & \xi<\xi_{1} \\ 1-\mathrm{e}^{\left(\xi-\xi_{3}\right) / c} & \xi_{1} \leq \xi \leq \xi_{3} \\ 0 & \xi>\xi_{3}\end{cases}
$$

We further restrict our attention to traveling pulse solutions where $q(\xi)>p(\xi)$ for $\xi \in\left(\xi_{2}, \xi_{4}\right)$, and $q(\xi)<p(\xi)$ otherwise, with $\xi_{1}<\xi_{2}<\xi_{3}<\xi_{4}$. In this case 28) 


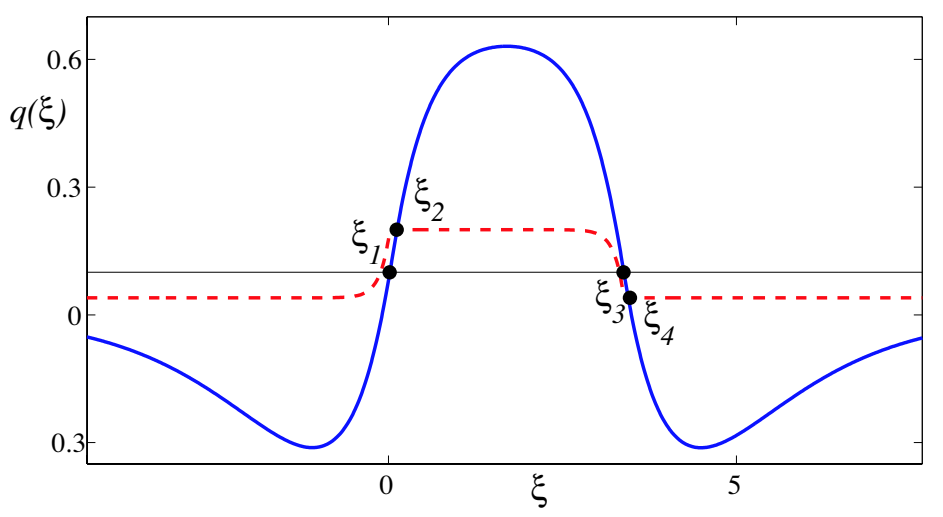

FiguRE 11. Analytical traveling pulse solution $(q, p)$ (as solid and dashed lines respectively) with $h_{0}=0.04 \theta=0.1, \kappa=0.16$ and $\alpha=1.6$.

takes the simple form

$$
\begin{aligned}
\psi(\xi) & =\int_{\xi_{2}-\xi}^{\xi_{4}-\xi} \mathrm{d} y w(y) \\
& = \begin{cases}g\left(\xi_{4}-\xi\right)-g\left(\xi_{2}-\xi\right) & \xi<\xi_{2} \\
g\left(\xi-\xi_{2}\right)+g\left(\xi_{4}-\xi\right) & \xi_{2} \leq \xi \leq \xi_{4} . \\
g\left(\xi-\xi_{2}\right)-g\left(\xi-\xi_{4}\right) & \xi>\xi_{4}\end{cases}
\end{aligned}
$$

Hence the solution for $q(\xi)$ is parameterized by the five unknowns $\xi_{1}, \xi_{2}, \xi_{3}, \xi_{4}, c$. By choosing an origin such that $\xi_{1}=0$ the simultaneous solution of the four threshold crossing conditions

$$
q\left(\xi_{1}\right)=\theta, \quad q\left(\xi_{2}\right)=p\left(\xi_{2}\right), \quad q\left(\xi_{3}\right)=\theta, \quad q\left(\xi_{4}\right)=p\left(\xi_{4}\right),
$$

may be used to determine the remaining four unknowns. For convenience we produce the functions $q\left(\xi_{i}\right)$ in the appendix. The numerical solution of $(33)$ shows that stable traveling pulses co-exist with stable bump solutions for a wide range of parameter values.

3.1. Evans function for a traveling pulse. Linearising (25) and (26) about the traveling wave solution and seeking solutions of the form $(\widetilde{u}(\xi, t), \widetilde{h}(\xi, t))=$ $(u(\xi), h(\xi)) \mathrm{e}^{\lambda t}$ gives

$$
\begin{aligned}
& u(\xi)=\frac{\left[u\left(\xi_{2}\right)-h\left(\xi_{2}\right)\right]}{\left|F^{\prime}\left(\xi_{2}\right)\right|} \mathcal{U}\left(\xi_{2}-\xi, \lambda\right)+\frac{\left[u\left(\xi_{4}\right)-h\left(\xi_{4}\right)\right]}{\left|F^{\prime}\left(\xi_{4}\right)\right|} \mathcal{U}\left(\xi_{4}-\xi, \lambda\right), \\
& h(\xi)=\frac{u\left(\xi_{1}\right)}{\left|q^{\prime}\left(\xi_{1}\right)\right|} \mathcal{H}\left(\xi_{1}-\xi, \lambda\right)+\frac{u\left(\xi_{3}\right)}{\left|q^{\prime}\left(\xi_{3}\right)\right|} \mathcal{H}\left(\xi_{3}-\xi, \lambda\right),
\end{aligned}
$$

where $F(s)=q(s)-p(s)$ and

$$
\begin{aligned}
\mathcal{U}(\xi, \lambda) & =\int_{0}^{\infty} \mathrm{d} y w(y-\xi) \eta(y / c) \mathrm{e}^{-\lambda y / c}, \\
c \mathcal{H}(\xi, \lambda) & =\kappa \eta_{h}(\xi / c) \mathrm{e}^{-\lambda \xi / c}=\kappa \mathrm{e}^{-(1+\lambda) \xi / c} H(\xi) .
\end{aligned}
$$


For an exponential synaptic response the integral in (36) gives:

$$
\begin{aligned}
\mathcal{U}(\xi, \lambda) & =\frac{\alpha}{c} \frac{\alpha+\lambda}{c} \mathrm{e}^{-(\alpha+\lambda) \xi / c}\left\{\frac{1}{[1+(\alpha+\lambda) / c]^{2}}-\frac{1}{[1-(\alpha+\lambda) / c]^{2}}\right\} \\
& +\frac{\alpha}{c} \frac{\mathrm{e}^{-\xi}}{1-(\alpha+\lambda) / c}\left\{\xi-1+\frac{1}{1-(\alpha+\lambda) / c}\right\}
\end{aligned}
$$

for $\xi \geq 0$ and

$$
\mathcal{U}(\xi, \lambda)=\frac{\alpha}{c} \frac{\mathrm{e}^{\xi}}{1+(\alpha+\lambda) / c}\left\{1+\xi-\frac{1}{1+(\alpha+\lambda) / c}\right\},
$$

for $\xi<0$. The derivatives $q^{\prime}$ and $F^{\prime}$ are easily calculated to give

$$
\begin{gathered}
q^{\prime}\left(\xi_{1}\right)=\alpha\left(\theta-\psi\left(\xi_{1}\right)\right) / c, \quad q^{\prime}\left(\xi_{3}\right)=\alpha\left(\theta-\psi\left(\xi_{3}\right)\right) / c, \\
F^{\prime}\left(\xi_{2}\right)=\left(\alpha\left(p\left(\xi_{2}\right)-\psi_{2}\right)-p\left(\xi_{2}\right)+h+\kappa\right) / c, \\
F^{\prime}\left(\xi_{4}\right)=\left(\alpha\left(p\left(\xi_{4}\right)-\psi\left(\xi_{4}\right)\right)-p\left(\xi_{4}\right)+h\right) / c .
\end{gathered}
$$

It is convenient to introduce the vector $z=\left(z_{1}, z_{2}, z_{3}, z_{4}\right)=\left(u\left(\xi_{1}\right), u\left(\xi_{2}\right)-\right.$ $\left.h\left(\xi_{2}\right), u\left(\xi_{3}\right), u\left(\xi_{4}\right)-h\left(\xi_{4}\right)\right)$. In which case we may obtain a set of matching conditions from (34) which we write as

$$
z_{i}=\sum_{j=2}^{4} A_{j}\left(\xi_{i}, \lambda\right) z_{j}, \quad i=2,3,4
$$

Here,

$$
\begin{aligned}
& A_{2}(\xi, \lambda)=\frac{\mathcal{U}\left(\xi_{2}-\xi, \lambda\right)}{\left|F^{\prime}\left(\xi_{2}\right)\right|}, \quad A_{3}(\xi, \lambda)=-\frac{\left.\mathcal{H}\left(\xi_{3}-\xi\right), \lambda\right)}{\left|q^{\prime}\left(\xi_{3}\right)\right|}, \\
& A_{4}(\xi, \lambda)=\frac{\mathcal{U}\left(\xi_{4}-\xi, \lambda\right)}{\left|F^{\prime}\left(\xi_{4}\right)\right|}
\end{aligned}
$$

Demanding that there be a nontrivial solution to 43 generates the eigenvalue problem $\mathcal{E}(\lambda)=|I-\mathcal{A}(\lambda)|=0$, where the $3 \times 3$ matrix $\mathcal{A}(\lambda)$ has components $[\mathcal{A}(\lambda)]_{i j}=A_{j}\left(\xi_{i}, \lambda\right)$.

Differentiation of the form for $\mathcal{U}(\xi, \lambda)$ and re-integrating between $\xi_{i}$ and $\xi$ gives us the useful result

$$
\mathcal{U}(\xi, \lambda)=\mathrm{e}^{-(\alpha+\lambda)\left(\xi-\xi_{i}\right) / c}\left[\mathcal{U}\left(\xi_{i}, \lambda\right)+\frac{\alpha}{c} \int_{0}^{\xi-\xi_{i}} w\left(s+\xi_{i}\right) \mathrm{e}^{(\alpha+\lambda) s / c} \mathrm{~d} s\right] .
$$

This allows us to re-write $u(\xi)=A_{2}(\xi, \lambda) z_{2}+A_{4}(\xi, \lambda) z_{4}$ as

$$
\begin{aligned}
u(\xi) & =\mathrm{e}^{(\alpha+\lambda)\left(\xi-\xi_{i}\right) / c}\left\{u\left(\xi_{i}\right)-\frac{\alpha}{c} \frac{z_{2}}{\left|F^{\prime}\left(\xi_{2}\right)\right|} \int_{0}^{\xi-\xi_{i}} w\left(\xi_{2}-\xi_{i}-s\right) \mathrm{e}^{-(\alpha+\lambda) s / c} \mathrm{~d} s\right. \\
& \left.-\frac{\alpha}{c} \frac{z_{4}}{\left|F^{\prime}\left(\xi_{4}\right)\right|} \int_{0}^{\xi-\xi_{i}} w\left(\xi_{4}-\xi_{i}-s\right) \mathrm{e}^{-(\alpha+\lambda) s / c} \mathrm{~d} s\right\} .
\end{aligned}
$$

Similarly we may obtain an expression for $h(\xi)$ as

$$
h(\xi)=\mathrm{e}^{(1+\lambda)\left(\xi-\xi_{i}\right) / c}\left\{h\left(\xi_{i}\right)-\frac{z_{1}}{\left|q^{\prime}\left(\xi_{1}\right)\right|} \mathcal{H}\left(\xi_{1}-\xi_{i}, \lambda\right)-\frac{z_{3}}{\left|q^{\prime}\left(\xi_{3}\right)\right|} \mathcal{H}\left(\xi_{3}-\xi_{i}, \lambda\right)\right\} .
$$

Restricting attention to the regime where $\operatorname{Re} \lambda>\max (-\alpha,-1)$ (i.e. to the right of the essential spectrum) we see from (46) and (47) that for $(u(\xi), h(\xi))$ to be 

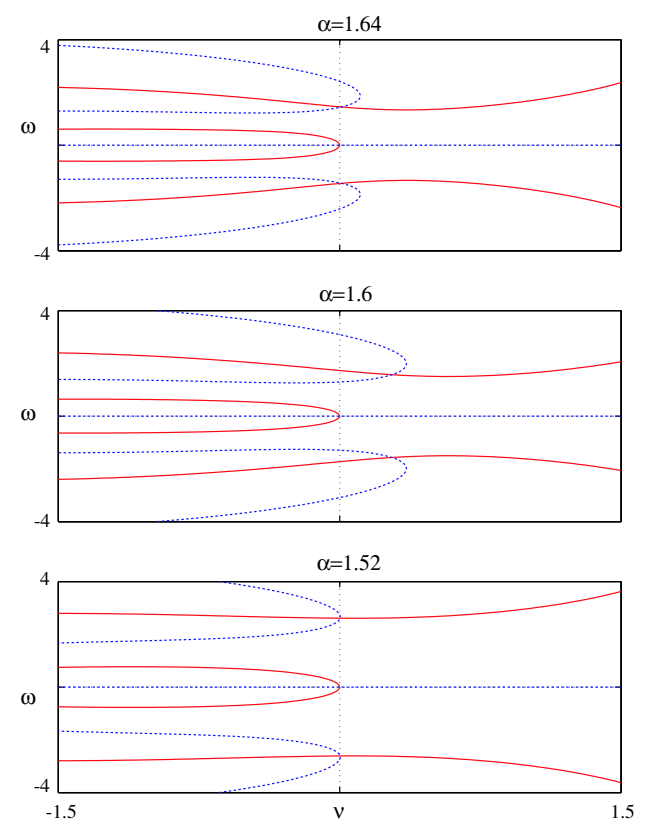

FIGURE 12. A plot of the Evans function for a traveling pulse at $\kappa=0.75$, and other parameters as in Fig. 1. A pulse can undergo a dynamic instability with increasing $\alpha$ and then restabilize via the reverse mechanism.

bounded as $\xi-\xi_{i} \rightarrow \infty$ we require the expressions in curly brackets to vanish. This generates a set of equations which is exactly equivalent to the matching conditions defined by 43 (and the further equation $\left.z_{1}=\sum_{j=2}^{4} A_{j}\left(\xi_{1}, \lambda\right) z_{j}\right)$. Thus, we interpret $\mathcal{E}(\lambda)$ as the Evans function of the traveling wave $(q(\xi), p(\xi))$. A straight forward calculation establishes that $(u, h)=\left(q^{\prime}, p^{\prime}\right)$ is an eigenfunction with $\lambda=0$ as expected.

Using the Evans function above to determine stability we find that a pulse can undergo a dynamic instability with increasing $\alpha$ and then restabilize via the reverse mechanism, see Fig. 12. Direct numerical simulations in such parameter windows show the emergence of stable traveling breathers. We illustrate this phenomenon in Fig. [13, and note that similar solutions have recently been observed in a reactiondiffusion system with subcritical wave instability [49]. Although such behavior may well be generic in inhomogeneous neural field models with external forcing, as in the work of Bressloff et al. [8, the appearance of a stable traveling breather in a homogeneous neural field model is a direct consequence of nonlinear threshold accommodation and does not occur in its absence.

\section{Exotic solutions and dissipative solitons}

The emergence of stable traveling breathers is not the only type of exotic behavior that can emerge in a network with nonlinear threshold accommodation. Direct numerical simulations show a whole host of exotic solutions including asymmetric breathers, multiple bumps, multiple pulses, periodic traveling waves, and 


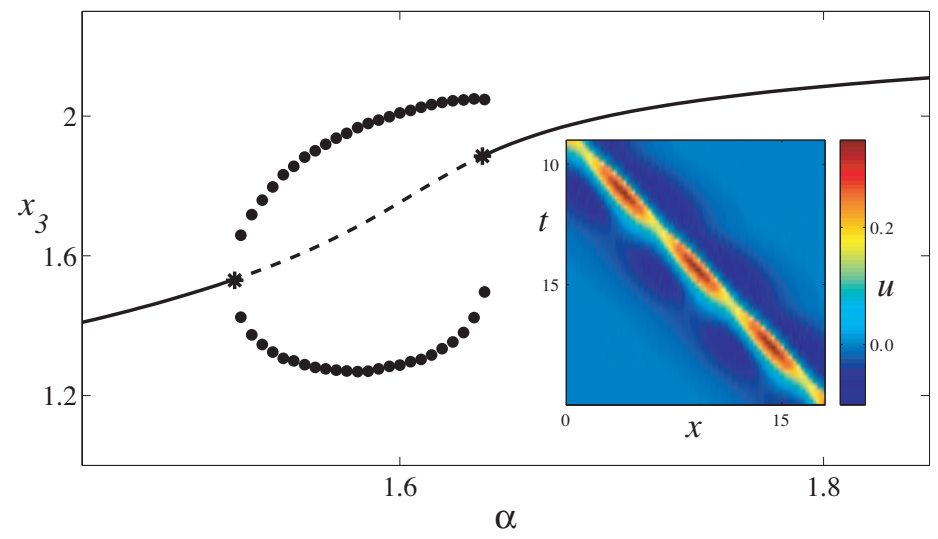

Figure 13. Analytically determined width $x_{3}$ of a traveling pulse solution as a function of the synaptic rate constant $\alpha$ (solid line is stable, dashed is unstable). As $\alpha$ increases through $\alpha \sim 1.52$ the Evans function shows that a pair of complex-conjugate eigenvalues crosses to the right hand complex plane. At $\alpha \sim 1.64$ they cross back to the left hand complex plane. Fig. 12 shows the Evans function at both these bifurcations. This leads to a branch of traveling breather solutions whose width oscillates between the indicated maximum and minimum values (circles). The inset shows an example of such a traveling breather, at $\alpha=1.58$.

bump-splitting instabilities that appear to lead to spatio-temporal chaos. An example of a such a splitting is shown in Fig. 14. Here we evolve bump-like initial data in a parameter regime where 1-bumps are known not to exist (i.e. to the right of the vertical dashed line in Fig. 7). It is interesting to note that similar bifurcations have been seen in other dissipative systems that support localized structures, in particular those of coupled cubic complex Ginzburg-Landau equations 42. Self-replication of pulses has also been observed in excitable reactiondiffusion systems, leading to the emergence of a self-similar Sierpinski gasket like structures in space-time plots [22, 23, 24. For the case of the Gray-Scott model Nishiura and Ueyama have proposed that such self-replicating patterns can be understood in terms of (ghosts of) limit points of folding bifurcation branches in parameter regions where the branches have ceased to exist [30, 36]. It remains an open problem to properly understand the bump-splitting seen here and to ascertain whether there is a true transition to spatio-temporal chaos, as seen for the Gray-Scott model [37. However, it is likely that an exploration of the hierarchy of limit points for the existence of $N$-bumps would provide a similar means for understanding the dynamics of self replicating bumps and spatio-temporal chaos in a neural network model with nonlinear threshold accommodation.

With the aid of direct numerical simulations we have also explored the scattering properties of traveling pulses in one dimension and traveling spots in two dimensions. This has led us to the fascinating observation that such structures can behave as quasi-particles in the sense that they can scatter like dissipative solitons 1, 28. Indeed, once again we see similarities of behavior to not only that of the Gray-Scott model, but to that of some three component reaction-diffusion systems 


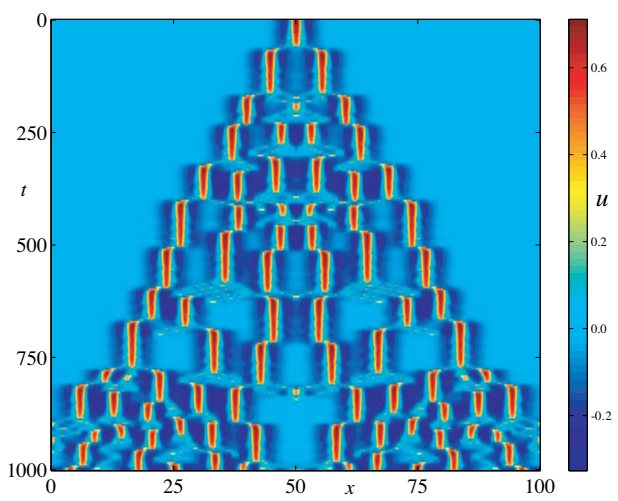

FiguRE 14. An example of a self-replicating bump in a regime where single bumps do not exist. Parameters are $h_{0}=0.02, \theta=$ $0.1, \kappa=0.34$ and $\alpha=0.5$.

[5, 45. An example of the scattering of two traveling pulses in one dimension is shown in Fig. 15. It is also possible that colliding pulses may entrain and move as a traveling two-pulse. Furthermore, for certain parameter values colliding pulses can annihilate one another, as shown in Fig. 16.
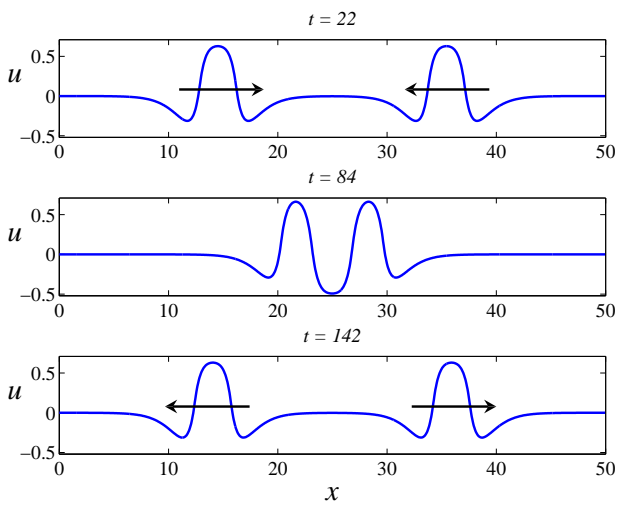

FIgURE 15. A symmetric pair of traveling pulses is used to prepare initial data on a large domain. As they travel toward each other the waves slow down before colliding and then ultimately reversing their direction. During the collision process the shape of the two waves is not preserved, though after scattering the traveling pulse shapes can once again be clearly identified. Parameters are $h_{0}=0.04, \theta=0.1, \kappa=0.16$ and $\alpha=1.58$.

It has been suggested by Nishiura et al. 35 that the scattering of dissipative solitons may be understood in terms of a so-called twin-horn separator. This is a special type of unstable solution that links input to output at collision. It is possible that such ideas may also be used to understand scattering in this neural 


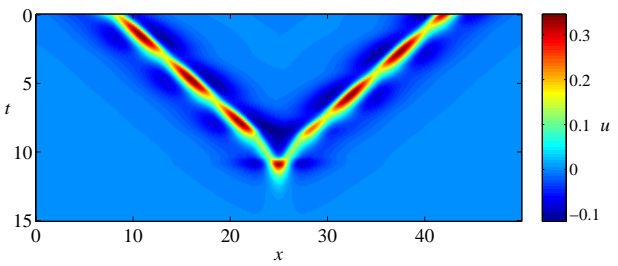

FiguRE 16. A pair of traveling breathing pulses travel toward each other, collide, and destroy one-another. In this case the relative initial phase of breathing oscillation makes no difference to the outcome. Parameters as for the inset in Fig. 13 .

model, though it is not obvious which unstable steady-state solution could play the role of the twin-horn separator ${ }^{2}$

As expected all of the solutions we have described in one space dimension have their analogs in a planar neural field model with nonlinear threshold accommodation. In Fig. 17 we show an example of two traveling spots scattering in a two dimensional domain. Initial data is a symmetric pair of spots traveling obliquely toward one another, and we see a repulsive interaction where the spots remain widely separated. For the same parameter set, but with the initial traveling spots oriented such that they head directly toward one another, Fig. 18 shows an example of temporary binding before separation. If the collision is not quite head-on, simulations indicate that a rotating bound state is possible, which ultimately decays to zero, as in Fig. 19
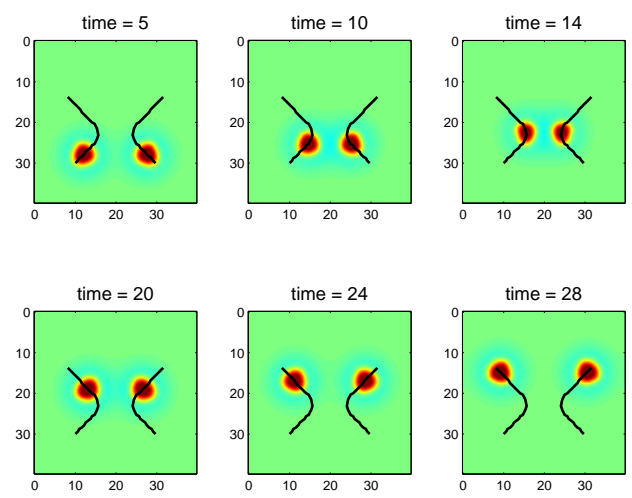

FiguRE 17. The scattering of a pair of traveling spots in a two dimensional domain (with toroidal geometry), showing a repulsive interaction where the spots remain widely separated. Parameters $\kappa=0.1, \theta=0.1, \alpha=2, h_{0}=0.08$.

Note that in the case of breathing solutions the phase may also play a role in how quasi-particles interact [46. It would be of obvious interest to develop a theory of quasi-particle interactions by deriving equations of motion for the interface (where $u$ crosses threshold), along the lines for reaction-diffusion models

\footnotetext{
${ }^{2}$ It is possible that the unstable 2-bump solution described in Section 2.2 could play this role. However, in simulations pulses can scatter even when that solution does not exist.
} 

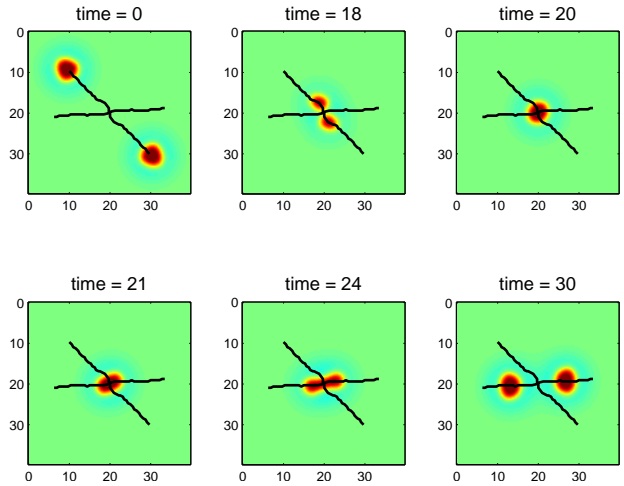

Figure 18. The collision of a pair of traveling spots in a two dimensional domain (with toroidal geometry), showing temporary binding before separation. Parameters as in Fig. 17.
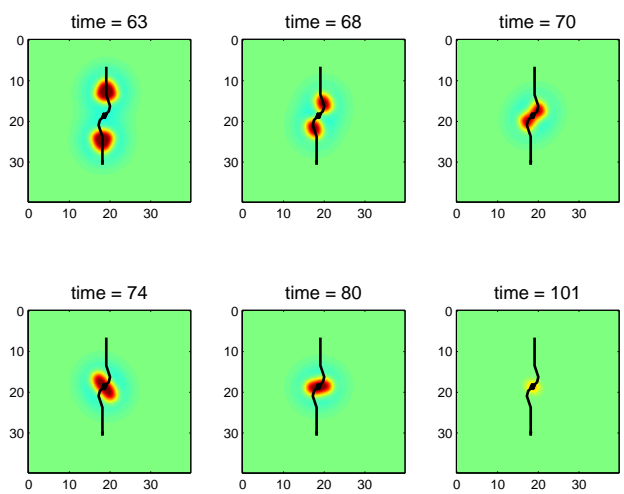

Figure 19. The collision of a pair of traveling spots in a two dimensional domain (with toroidal geometry). After collision one sees a rotating bound state which ultimately decays to zero. Parameters as in Fig. 17.

described in 27, 39. Alternatively one may consider deriving equations of motion for quasi-particle centroids along the lines described by Elphick et al. [14 as recently done by Bode et al. [5] for a three-component reaction diffusion system and Bressloff [6] for neural field models. In this latter approach one must assume weak interaction between quasi-particles (so that their shape is preserved during collision), as in the example of Fig. 17. However, shapes may not be preserved, as exemplified by Figs. 18 and 19 .

The mathematical machinery for describing radially symmetric bumps can be built up in a similar way to that developed for the one dimensional model 18. Interestingly, even in the absence of threshold accommodation $(\kappa=0)$, a 1-bump can undergo an instability with decreasing $h_{0}$ at the point where the center of the bump develops a point of inflection (or dimple). In this case an Evans function approach shows that the bump can split into two pieces $\mathbf{9}$. For the interested reader a fuller exposition of bumps, rings and their instabilities in planar neural field models can be found in 40. However, with the inclusion of 
threshold accommodation such an instability can lead to patterning of the neural medium post bifurcation. An example of this is shown in Fig. 20
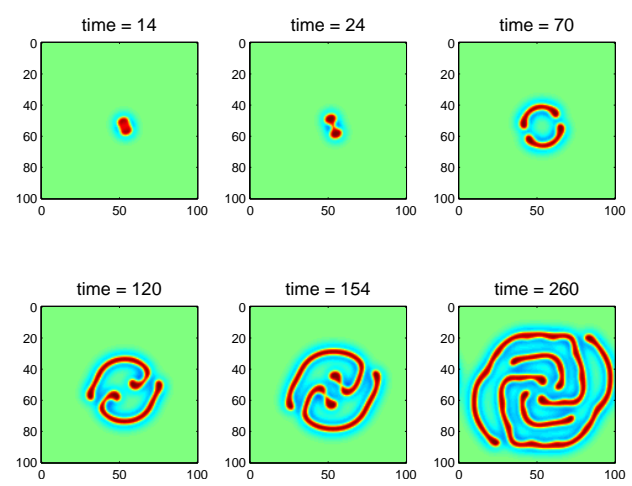

Figure 20. After a (dimple) bump goes unstable in a planar neural field model with threshold accommodation one typically sees the emergence of a pattern state as illustrated here. Parameters $h_{0}=0.06, \theta=0.1, \kappa=0.05, \alpha=1.0$. 


\section{Discussion}

In this paper we have developed a tractable formulation of threshold accommodation for neural-field theories, motivated by the ideas of A V Hill in 1936 25. Hill observed that the threshold for neural excitation should be dependent upon the state of the tissue, and in particular should rise when the local potential is maintained at a high level, and otherwise revert to its resting level. Our phenomenological model therefore includes a dynamically varying threshold whose basal level $h_{0}$ is driven toward $h_{0}+\kappa$ when the synaptic activity $u$ (identified here with the "local potential" described by Hill) is elevated above a threshold $\theta$.

We have focused on connectivity with short-range excitation and long range inhibition (the so-called Mexican-hat), which can support spatially localized solutions without accommodation. We chose to work with the Heaviside firing rate function in order to facilitate analytical progress. We used Evans function techniques to study instabilities of spatially localized states in one space dimension, and showed that breathing bumps and pulses can emerge as instabilities of the basic single bump solution. Furthermore, we demonstrated, again using the Evans function, that pulse solutions themselves can undergo a dynamic instability in favor of breathing pulse solutions. These novel breathing instabilities represent interesting new dynamics solely due to the inclusion of nonlinear threshold accommodation. Interestingly, numerical simulations show that nonlinear threshold accommodation leads to a variety of more exotic solutions, including bump splitting, scattering and annihilation in both one and two space dimensions. To understand these complex behaviors is an open challenge. 


\section{Appendix}

It is straight forward (though laborious) to calculate $q(\xi)$ of Section 3 in closed form using (27) and (32). However, we only need to evaluate $q(\xi)$ at four specific points. Performing these calculations for the choice of an exponential synapse gives

$$
\begin{aligned}
& q\left(\xi_{1}\right)=\frac{\alpha}{c} \mathrm{e}^{\alpha \xi_{1} / c}\left[\phi\left(\xi_{1}, \xi_{2}\right)+\phi\left(\xi_{2}, \xi_{4}\right)+\phi\left(\xi_{4}, \infty\right)\right], \\
& q\left(\xi_{2}\right)=\frac{\alpha}{c} \mathrm{e}^{\alpha \xi_{2} / c}\left[\phi\left(\xi_{2}, \xi_{4}\right)+\phi\left(\xi_{4}, \infty\right)\right], \\
& q\left(\xi_{3}\right)=\frac{\alpha}{c} \mathrm{e}^{\alpha \xi_{3} / c}\left[\phi\left(\xi_{3}, \xi_{4}\right)+\phi\left(\xi_{4}, \infty\right)\right], \\
& q\left(\xi_{4}\right)=\frac{\alpha}{c} \mathrm{e}^{\alpha \xi_{4} / c} \phi\left(\xi_{4}, \infty\right),
\end{aligned}
$$

where

$$
\phi(a, b)=\int_{a}^{b} \mathrm{~d} s \psi(s) \mathrm{e}^{-\alpha s / c} .
$$

The explicit forms of $\phi\left(\xi_{1}, \xi_{2}\right)$ etc. are most easily calculated using the result that

$$
g(x)=-\left.\frac{\mathrm{d}}{\mathrm{d} \beta} \mathrm{e}^{-\beta x}\right|_{\beta=1} .
$$

The expressions in (48) to (51) may then be compactly represented as $\phi\left(\xi_{1}, \xi_{2}\right)=$ $-\Phi_{12}^{\prime}(1)$ etc. where

$$
\begin{aligned}
& \Phi_{12}(\beta)=\frac{\mathrm{e}^{-(\alpha / c-\beta) \xi_{1}}-\mathrm{e}^{-(\alpha / c-\beta) \xi_{2}}}{\alpha / c-\beta}\left[\mathrm{e}^{-\beta \xi_{4}}-\mathrm{e}^{-\beta \xi_{2}}\right] \\
& \Phi_{24}(\beta)=\frac{\mathrm{e}^{-(\alpha / c+\beta) \xi_{2}}-\mathrm{e}^{-(\alpha / c+\beta) \xi_{4}}}{\alpha / c+\beta} \mathrm{e}^{\beta \xi_{2}}+\frac{\mathrm{e}^{-(\alpha / c-\beta) \xi_{2}}-\mathrm{e}^{-(\alpha / c-\beta) \xi_{4}}}{\alpha / c-\beta} \mathrm{e}^{-\beta \xi_{4}}, \\
& \Phi_{34}(\beta)=\frac{\mathrm{e}^{-(\alpha / c+\beta) \xi_{3}}-\mathrm{e}^{-(\alpha / c+\beta) \xi_{4}}}{\alpha / c+\beta} \mathrm{e}^{\beta \xi_{2}}+\frac{\mathrm{e}^{-(\alpha / c-\beta) \xi_{3}}-\mathrm{e}^{-(\alpha / c-\beta) \xi_{4}}}{\alpha / c-\beta} \mathrm{e}^{-\beta \xi_{4}},
\end{aligned}
$$

$\Phi_{4 \infty}(\beta)=\frac{\mathrm{e}^{-(\alpha / c+\beta) \xi_{4}}}{\alpha / c+\beta}\left[\mathrm{e}^{\beta \xi_{2}}-\mathrm{e}^{\beta \xi_{4}}\right]$.

\section{References}

1. N Akhmediev and A Ankiewicz (eds.), Dissipative Solitons, Lecture Notes in Physics, no. 661, Springer, 2005.

2. S Amari, Homogeneous nets of neuron-like elements, Biological Cybernetics 17 (1975), 211220.

3. R Ben-Yishai, L Bar-Or, and H Sompolinsky, Theory of orientation tuning in visual cortex, Proceedings of the National Academy of Sciences USA 92 (1995), 3844-3848.

4. R L Beurle, Properties of a mass of cells capable of regenerating pulses, Philosophical Transactions of the Royal Society London B 240 (1956), 55-94.

5. M Bode, A W Liehr, C P Schenk, and H G Purwins, Interaction of dissipative solitons: partile-like behaviour of localized structures in a three-component reaction-diffusion system, Physica D 161 (2002), 45-66.

6. P C Bressloff, Weakly-interacting pulses in synaptically coupled neural media, SIAM Journal on Applied Mathematics 66 (2005), 57-81. 
7. P C Bressloff, J D Cowan, M Golubitsky, P J Thomas, and M Wiener, Geometric visual hallucinations, Euclidean symmetry and the functional architecture of striate cortex, Philosophical Transactions of the Royal Society London B 40 (2001), 299-330.

8. P C Bressloff, S E Folias, A Prat, and Y X Li, Oscillatory waves in inhomogeneous neural media, Physical Review Letters 91 (2003), 178101.

9. S Coombes, Waves, bumps, and patterns in neural field theories, Biological Cybernetics 93 (2005), 91-108.

10. , Neural fields, Scholarpedia (2006), 2125.

11. S Coombes, G J Lord, and M R Owen, Waves and bumps in neuronal networks with axodendritic synaptic interactions, Physica D 178 (2003), 219-241.

12. S Coombes and M R Owen, Evans functions for integral neural field equations with Heaviside firing rate function, SIAM Journal on Applied Dynamical Systems 34 (2004), 574-600.

13. _ Bumps, breathers, and waves in a neural network with spike frequency adaptation, Physical Review Letters 94 (2005), 148102(1-4).

14. C Elphick, E Meron, and E A Spiegel, Patterns of propagating pulses, SIAM Journal on Applied Mathematics 50 (1990), 490-503.

15. G B Ermentrout and J D Cowan, A mathematical theory of visual hallucination patterns, Biological cybernetics 34 (1979), 137-150.

16. J Evans, Nerve axon equations: IV The stable and unstable impulse, Indiana University Mathematics Journal 24 (1975), 1169-1190.

17. S Folias and P C Bressloff, Stimulus-locked traveling waves and breathers in an excitatory neural network, SIAM Journal on Applied Dynamical Systems 65 (2005), 2067-2092.

18. S E Folias and P C Bressloff, Breathing pulses in an excitatory neural network, SIAM Journal on Applied Dynamical Systems 3 (2004), 378-407.

19. M A Geise, Neural field theory for motion perception, Kluwer Academic Publishers, 1999.

20. J S Griffith, A field theory of neural nets: I: Derivation of field equations, Bulletin of Mathematical Biophysics 25 (1963), 111-120.

21. _ A field theory of neural nets: II: Properties of field equations, Bulletin of Mathematical Biophysics 27 (1965), 187-195.

22. Y Hayase and T Ohta, Sierpinski gasket in a reaction-diffusion system, Physical Review Letters 81 (1998), 1726-1729.

23. __ Self-replicating pulses and Sierpinski gaskets in excitable media, Physical Review E 62 (2000), 5998-6003.

24. $\quad$ Self-replication of a pulse in excitable reaction-diffusion systems, Physical Review E 66 (2002), 036218.

25. A V Hill, Excitation and accommodation in nerve, Proceedings of the Royal Society of London. Series B, Biological Sciences 119 (1936), 305-355.

26. X Huang, W C Troy, Q Yang, H Ma, C R Laing, S J Schiff, and J Wu, Spiral waves in disinhibited mammalian neocortex, The Journal of Neuroscience 24 (2004), 9897-9902.

27. S Kawaguchi and M Mimura, Collision of travelling waves in a reaction-diffusion system with global coupling effect, SIAM Journal on Applied Mathematics 59 (1999), 920-941.

28. B S Kerner and V V Osipov, Autosolitons, Fundamental Theories of Physics, vol. 61, Kluwer Academic Publishers, 1994

29. K Kishimoto and S Amari, Existence and stability of local excitations in homogeneous neural fields, Journal of Mathematical Biology 7 (1979), 303-318.

30. T Kolokolnikov, M J Ward, and J Wei, The existence and stability of spike equilibria in the one-dimensional Gray-Scott model: The pulse-splitting regime, Physica D 202 (2005), 258-293.

31. P I Nunez, Neocortical dynamics and human EEG rhythms, Oxford University Press, 1995.

32. C R Laing, W C Troy, B Gutkin, and G B Ermentrout, Multiple bumps in a neuronal model of working memory, SIAM Journal on Applied Mathematics 63 (2002), 62-97.

33. L Lapicque, Recherches quantitatives sur l'excitation électrique des nerfs traitée comme une polarisation, J. Physiol. Pathol. Gen. 9 (1907), 620-635.

34. D T J Liley, P J Cadusch, and M P Dafilis, A spatially continuous mean field theory of electrocortical activity, Network 13 (2002), 67-113

35. Y Nishiura, T Teramoto, and K-I Ueda, Scattering and separators in dissipative systems, Physical Review E 67 (2003), 056210.

36. Y Nishiura and D Ueyama, A skeleton structre of self-replicating dynamics, Physica D 130 (1999), 73-104.

37. __ Spatio-temporal chaos for the Gray-Scott model, Physica D 150 (2001), 137-162. 
38. P L Nunez, The brain wave equation: a model for the EEG, Mathematical Biosciences 21 (1974), 279-297.

39. T Ohta, Pulse dynamics in a reaction-diffusion system, Physica D 151 (2001), 61-72.

40. M R Owen, C R Laing, and S Coombes, Bumps, rings, and spots in a two-dimensional neural field: splitting and rotational instabilities, In preparation (2007).

41. D J Pinto, R K Jackson, and C E Wayne, Existence and stability of traveling pulses in a continuous neuronal network, SIAM Journal on Applied Dynamical Systems 4 (2005), 954-984.

42. H Sakaguchi and B A Malomed, Instabilities and splitting of pulses in coupled GinzburgLandau equations, Physica D 154 (2001), 229-239.

43. B Sandstede, Handbook of dynamical systems II, ch. Stability of travelling waves, pp. 9831055, Elsevier, 2002.

44. _ Evans functions and nonlinear stability of travelling waves in neuronal network models, International Journal of Bifurcation and Chaos to appear (2007).

45. C P Schenk, M Or-Guil, M Bode, and H-G Purwins, Interacting pulses in three-component reaction-diffusion systems on two-dimensional domains, Physica Review Letters 78 (1997), $3781-3784$

46. T Teramoto, K-I Ueda, and Y Nishiura, Phase-dependent output of scattering process for traveling breathers, Physica Review E 69 (2004), 056224.

47. E L White, Cortical circuits: Synaptic organization of the cerebral cortex. Structure, function, and theory, Birkhauser, 1989.

48. H R Wilson and J D Cowan, Excitatory and inhibitory interactions in localized populations of model neurons, Biophysical Journal 12 (1972), 1-24.

49. L Yang, A M Zhabotinsky, and I R Epstein, Jumping solitary waves in an autonomous reaction-diffusion system with subcritical wave instability, Physical Chemistry Chemical Physics 8 (2006), 4647-4651.

50. K Zhang, Representation of spatial orientation by the intrinsic dynamics of the headdirection cell ensemble: a theory, Journal of Neuroscience 16 (1996), 2112-2126.

51. L Zhang, On stability of traveling wave solutions in synaptically coupled neuronal networks, Differential and Integral Equations 16 (2003), 513-536.

School of Mathematical Sciences, University of Nottingham, Nottingham, NG7 2RD, UK.

E-mail address: stephen.coombes@nottingham.ac.uk

$U R L:$ http://www. maths.nott.ac.uk/ sc/

E-mail address: Markus.0wen@nottingham.ac.uk

URL: http://www . maths.nott.ac.uk/ mro/ 